\title{
Influence of Aging, Sterilization, and Composition on the Degradation of Polyurethane Foams
}

Sam T Briggs ${ }^{1}$, Mary Beth B Monroe ${ }^{3}$, Mark A Wierzbicki ${ }^{1}$, Sayyeda Marziya Hasan ${ }^{2}$, Duncan J Maitland ${ }^{1,2, *}$

1. Biomedical Engineering, Texas A\&M University, College Station, Texas 77843, USA; E-Mails: sambriggs@tamu.edu; mwierzbicki03@gmail.com; djmaitland@tamu.edu

2. Shape Memory Medical, Inc., Santa Clara, CA 95054, USA; E-Mail: marziya@shapemem.com

3. Biomedical and Chemical Engineering, Syracuse University, Syracus, New York 13244, USA; E-mail: mbmonroe@syr.edu

* Correspondence: Duncan Maitland; E-Mail: djmaitland@tamu.edu

Academic Editor: Hossein Hosseinkhani

Recent Progress in Materials

2021, volume 3, issue 2

doi:10.21926/rpm.2102025
Received: April 05, 2021

Accepted: June 04, 2021

Published: June 25, 2021

\begin{abstract}
Shape memory polymers (SMPs) are highly attractive materials for medical devices. Specifically, SMP foams are currently being used as embolic devices in peripheral and cerebral vascular applications. To ensure the proper function and safety of these materials in their intended applications, it is important to understand how processing treatments, such as aging, sterilization, and foam composition can influence their degradation. Here, SMP foams were treated with industry relevant processing parameters, and the influence on degradation was observed via gravimetric, chemical, and morphological studies. Accelerated thermal aging was shown to have an influence on material degradation rate in real time oxidative studies. Sterilization was performed via Electron beam (E-beam) irradiation at the high and low dosages commonly used in industry and had no significant influence on foam degradation profiles. These findings help inform appropriate treatment of SMP foam embolic devices to ensure their safety and efficacy.
\end{abstract}

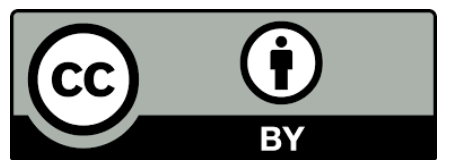

(C) 2021 by the author. This is an open access article distributed under the conditions of the Creative Commons by Attribution License, which permits unrestricted use, distribution, and reproduction in any medium or format, provided the original work is correctly cited. 


\section{Keywords}

Degradation; aging; sterilization; shape memory

\section{Introduction}

Degradation is a vital consideration in the design of any implantable medical device. If the material degrades too quickly, it can fail to provide required mechanical support or produce high concentrations of cytotoxic by-products [1, 2]. If it degrades too slowly, it may inhibit replacement or ingrowth by native tissues. For this reason, much work goes into understanding the degradation profile of biomaterials used in medical devices. Many factors can influence a material's degradation profile in ways that can alter in vivo performance, including processing conditions, sterilization, and handling and storage. Understanding the influence of these factors on the degradation profile is required to achieve clinical success of implanted materials [3].

Polyurethanes have several beneficial characteristics for biomedical applications, such as biocompatibility, strength, and processability [4]. They have been used in devices ranging from catheters to pacemaker leads and continue to be studied for use in novel devices. Additionally, certain polyurethanes can show a shape memory effect [5]. Shape-memory polymers (SMPs) can be fabricated in a primary shape that can be deformed to a secondary shape, typically while heated above a transition temperature. If cooled while deformed, the material will hold this secondary shape until it is again heated above the transition temperature, at which time a thermally induced shape-recovery takes place [6]. This shape memory ability can be beneficial for a number of applications ranging from biomaterials [7] to aerospace [8]. Recently, advances have been made in increasing Tg of SMPs with high recovery stress [9] and using shape memory composites for electromagnetic shielding [10].

Among SMP scaffolds, foams are of particular interest due to high levels of compressibility and volume recovery. Namely, SMP foams are particularly useful for minimally invasive procedures, wherein they can be compressed to a low-profile delivery shape, guided to the desired area in the body, and then expanded to their primary, clinically relevant shape once in place. Recently, SMP foams were approved for use as embolic devices in medical application (IMPEDE Embolization Plug, Shape Memory Medical, Inc.). These porous poly (amino urethane) foams form a tortuous pathway for blood, initiating the clotting cascade, and serving as a scaffold for stable clot formation [11]. Previous studies have investigated the in vitro and in vivo degradation profiles of SMP foams. These studies showed that the materials are resistant to hydrolytic degradation (though variations have been created to allow for hydrolysis) but are susceptible to oxidative degradation [11-13]. This degradation is believed to occur via oxidation of the tertiary amines in foaming monomers. None of these previous studies have investigated the influence of common processing parameters, such as sterilization via electron beam (E-beam) or aging, on SMP foam, both of which are important considerations for biomaterial commercialization.

One consideration with sterilizing SMP foams is their sensitivity to heat and moisture. For embolic applications, it is requisite that the materials are sterilized in the crimped, secondary state and that they are not actuated by the sterilization process. Thus, techniques that use high temperature or moisture, such as autoclaving and traditional ethylene oxide, are not viable. 
Previous studies with SMP foams compared the impact of a modified ethylene oxide (EtO) gas treatment and E-beam irradiation sterilization on thermomechanical properties [14]. They showed that even the lower temperatures and humidity of the modified EtO gas treatment, as compared to traditional EtO, influenced the glass transition temperature of the materials and caused premature expansion in the packaging. E-beam sterilization had minimal influence on the thermomechanical properties. Gamma radiation was not used for this study due to concerns regarding oxidative degradation [15].

E-beam irradiation is a popular technique for sterilizing medical devices, and ideal for SMP foams, because it can be performed in ambient temperatures and does not add any potentially cytotoxic chemicals. E-beam sterilization works by producing a concentrated beam of electrons that disrupts chemical bonds and DNA, disabling replication in cells and microbes [16]. This technique has a short penetration depth but is ideal for low density materials such as SMP foams. The dosage used for sterilization is generally between 20-30 kGy with the industry standard set at $25 \mathrm{kGy}$ (ISO 11137). Ebeam is also commonly used to form crosslinks in various polymers as it can alter chemical bonds in the polymer backbone, though the required dosages are generally much higher than those used for sterilization [17]. Even at low dosages, E-beam irradiation can influence the backbone structure of a polymer [18]. Murray et al. investigated the effect of E-beam irradiation ranging from $5 \mathrm{kGy}$ to 200 kGy on a commercial polyurethane (Pellethane 2363 90A) and found that even at dosages as low as $25 \mathrm{kGy}$, observable changes occurred in chemical structure as measured by Fourier transform infrared (FTIR) spectroscopy [19]. Kang et al. investigated the influence of E-beam sterilization on the in vivo degradation of $\beta$-tricalcium phosphate/polycaprolactone using volumetric microcomputed tomography measurements and found that sterilized samples degraded faster than nonsterilized samples [20]. However, no studies have been performed with SMP foams to determine if E-beam sterilization changes the material in a way that impacts the degradation profile.

Accelerated aging is a commonly used technique to determine the appropriate shelf life of a material. Often, physical changes in the material are measured throughout aging, such as colorimetric changes [21-24], changes in mechanical strength or integrity [25-32], or thermomechanical changes in the glass transition or melting temperatures [27,33]. Additionally, chemical changes can be measured using FTIR and/or mass spectroscopy. Accelerated aging is commonly achieved by storing the material at an elevated temperature. This process can be correlated to real-time aging based on the Arrhenius equation, which states that the chemical reaction rate increases with temperature [34]. Many industry standards are based on this equation, including AAMI TIR 17 and ASTM F1980, which use a reaction rate factor of Q10. Previous studies with SMP foams have investigated the effect of aging on the shape memory properties (strain fixity and recovery) [35]. However, gravimetric degradation studies have not been performed to ensure appropriate function of the device after aging.

In this study we investigate the influence of E-beam sterilization and aging on the degradation profile of SMP foams intended for occlusion applications. These studies aim to ensure the success and safety of these devices after sterilization and storage. 


\section{Materials and Methods}

\subsection{Materials}

$\mathrm{N}, \mathrm{N}, \mathrm{N}^{\prime}, \mathrm{N}^{\prime}$-tetrakis (2-hydroxypropyl)ethylenediamine (HPED, 99\%; Sigma-Aldrich Inc., St. Louis, MO, USA), triethanolamine (TEA, 98\%; Sigma-Aldrich Inc., St. Louis, MO, USA), trimethyl-1,6hexamethylene diisocyanate, 2,2,4- and 2,4,4- mixture (TMHDI, TCI America Inc., Portland, OR, USA), Vorasurf DC 198 (Dow Corning, Midland, MI, USA), Vorasurf DC 5943 (Dow Corning, Midland, MI, USA), DABCO T-131 (Evonik Industries AG, Essen, Germany), DABCO BL-22 (Evonik Industries AG, Essen, Germany), Ortegol 500 (Evonik Industries AG, Essen, Germany), and deionized (DI) water (ASTM Type II; LabChem, < 1 $\mu \mathrm{S} / \mathrm{cm}$ ) were used as received for foam fabrication. 2-propanol 99\% (IPA) (VWR, Radnor, PA, USA), was used for foam cleaning before sterilization and aging. Phosphate buffered saline (PBS), UPG Powder (Amresco Inc., Solon, $\mathrm{OH}$ ) and sodium hydroxide (Sigma-Aldrich Inc. St. Lous, $\mathrm{MO}$ ) were used for hydrolytic degradation studies. Hydrogen peroxide solution, $50 \mathrm{wt} \%$ in $\mathrm{H}_{2} \mathrm{O}$, stabilized (Sigma-Aldrich Inc., St. Louis, $\mathrm{MO}$ ) was diluted to the desired concentration ( $3 \%$ or $20 \%)$ in reverse osmosis water and stored at $-4{ }^{\circ} \mathrm{C}$.

\subsection{Foam Synthesis and Treatment}

SMP foams were synthesized using the multi-step protocol previously described by Hasan et al. [36]. Briefly, an isocyanate (NCO) pre-polymer was synthesized using the molar ratios of $60 \%$ HPED, 40\%TEA, and 100\% TMHDI (H60) or 70\% HPED, 30\%TEA, and 100\% TMHDI (H70), with a 43 wt\% hydroxyl $(\mathrm{OH})$ component. An $\mathrm{OH}$ mixture was prepared with the remaining weight percentage of HPED and TEA, along with catalysts (DABCO T-131 and DABCO BL-22), surfactants (Vorasurf DC198 and Vorasurf DC5943), cell opener (Ortegol 500), and DI water. DI water was used as a chemical blowing agent to generate carbon dioxide bubbles via a reaction with free isocyanates. The NCO premix and the $\mathrm{OH}$ component was mixed using a speedmixer (FlakTek Inc., Landrum, SC, USA) and the foam mixture was poured into a tray. The resulting foam was cured in a vacuum oven (Cascade Tek, Hillsboro, Oregon) at $90^{\circ} \mathrm{C}$ for 10 minutes. The SMP foam was cooled to room temperature (21 $\left.\pm 1{ }^{\circ} \mathrm{C}\right)$ followed by a 24 -hour cold cure $\left(21 \pm 1^{\circ} \mathrm{C}\right)$ before further processing. Table 1 shows the weight percent of each component used for foam synthesis and Figure 1 shows the chemical structure of the monomers used to fabricate the SMP network and a hypothetical schematic of the amorphous network.

Table 1 Weight percentage of monomers used during the foaming process.

\begin{tabular}{|c|c|c|c|c|c|c|c|c|c|}
\hline \multirow{2}{*}{$\begin{array}{l}\text { Foam } \\
\text { Composition }\end{array}$} & \multicolumn{9}{|c|}{ Weight Percent (wt \%) } \\
\hline & TMHDI & Ortegol 500 & $\mathrm{~T}-131$ & BL-22 & DC 198 & DC 5943 & HPED & TEA & Water \\
\hline \multicolumn{10}{|l|}{ 100TMHDI } \\
\hline HPED60 & 63.07 & 0.51 & 1.26 & 1.89 & 2.48 & 4.47 & 16.58 & 7.53 & 2.21 \\
\hline \multicolumn{10}{|l|}{ TEA40 } \\
\hline \multicolumn{10}{|l|}{ 100TMHDI } \\
\hline HPED70 & 63.73 & 0.53 & 0.51 & 0.64 & 2.52 & 4.55 & 19.55 & 5.71 & 2.25 \\
\hline TEA30 & & & & & & & & & \\
\hline
\end{tabular}




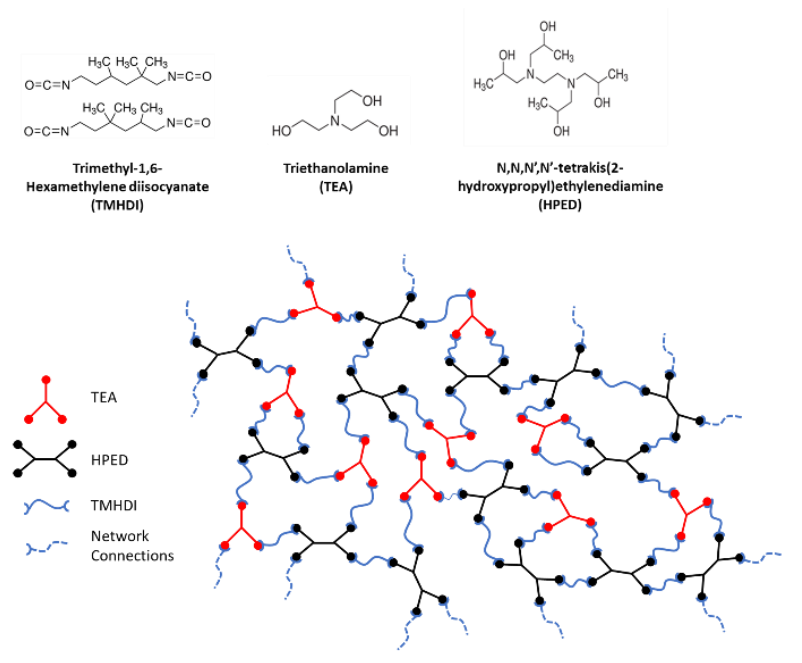

Figure 1 Molecular structure of the monomers used to create the SMP backbone and polymer network schematic.

Foam cubes $(1 \mathrm{~cm} \times 1 \mathrm{~cm} \times 1 \mathrm{~cm})$ were cut out of the bulk foam and cleaned in 1 litre glass jars using one 30-minute sonication wash in DI water, two 30 minutes sonication washes in $70 \%$ isopropyl alcohol (IPA), and four 30 minutes sonication washes in IPA. After each wash, the solvent was discarded, and the jars were replenished with fresh solvent. Prior to testing, foam cylinders were dried at $100{ }^{\circ} \mathrm{C}$ under vacuum for at least 12 hours after which they were stored in a plastic storage container with desiccant.

After cleaning, all foam cubes were packaged and sent to SteriTek (Fremont, CA, USA) and sterilized via electron beam at 21, 25, or $31 \mathrm{kGy}$. Foams to be aged were then sent to Westpak Inc. (San Jose, CA, USA) for 1-year accelerated aging. This accelerated aging process followed ASTM $F$ 1980-07 with an aging temperature of $55{ }^{\circ} \mathrm{C}$ for 40 days. The accelerated aging time (AAT) was calculated using the following formula, which is derived from the Arrhenius equation:

$$
\text { Accelerated Aging Time }(A A T)=\frac{\text { Desired Real Time }(R T)}{Q_{10}^{\left[\left(T_{A A}-T_{R T}\right) / 10\right]}}
$$

where RT is the desired real time, $T_{A A}$ is the accelerated aging temperature in ${ }^{\circ} \mathrm{C}, T_{R T}$ is the ambient temperature in ${ }^{\circ} \mathrm{C}$, and $Q_{10}$ is the aging factor. An aging temperature of $55^{\circ} \mathrm{C}$ was selected as this is the highest temperature to which the foams can be exposed before the onset of the material's glassy-to-rubbery state transition.

\subsection{Degradation and Gravimetric Analysis}

Oxidative degradation solutions were made by diluting the $50 \% \mathrm{H}_{2} \mathrm{O}_{2}$ in reverse osmosis water to the desired concentration ( $20 \% \mathrm{H}_{2} \mathrm{O}_{2}$ for accelerated analysis and $3 \% \mathrm{H}_{2} \mathrm{O}_{2}$ for real-time analysis). For the real time hydrolytic degradation solutions, PBS was prepared in RO water according to manufacturer instructions. Accelerated hydrolytic solutions $(0.1 \mathrm{M} \mathrm{NaOH})$ where made by dissolving $\mathrm{NaOH}$ pellets in $\mathrm{RO}$ water.

All sterilized foam cubes (aged and non-aged groups) were weighed upon arrival to obtain an initial weight. They were then placed in a labelled glass vial, submerged in $20 \mathrm{~mL}$ of the appropriate 
degradation solution, and incubated at $37{ }^{\circ} \mathrm{C}$. Solution levels were checked daily and solutions were changed every three days. Mass measurements were taken every 6 days for accelerated oxidative solutions, every 20 days for real time oxidative solutions, and every 14 days (28 days after day 70 ) for all hydrolytic samples. When taking mass measurements, the degradation solution was decanted while retaining the sample in the vial. $20 \mathrm{~mL}$ of ethanol was added to each vial and samples were allowed to sit in the ethanol for 3 minutes before the ethanol was decanted. Sample vials were covered with a laboratory wipe and dried in a vacuum oven at $50{ }^{\circ} \mathrm{C}$ for a minimum of 12 hours. Once dry, samples were removed from the oven and the mass was measured on a precision scale ( $1 \mathrm{mg}$ resolution). They were then returned to their respective vial and re-submerged in the degradation solution.

For the oxidative degradation samples, weighing was stopped when the samples broke apart and could no longer be removed from the vial without causing significant mechanical degradation or losing material.

\subsection{Morphological and Chemical Characterization}

Morphological changes were observed using scanning electron microscopy (SEM) images captured throughout the degradation process. For SEM images, a thin slice ( $1 \mathrm{~mm}$ ) was taken from a sacrificial sample and dried in an oven at $50{ }^{\circ} \mathrm{C}$. It was then seated on a metal stub with carbon black tape and sputter coated for 60 seconds using a Ted Pella Cressington 108 gold sputter coater (Ted Pella Inc., Redding, CA). Images were captured using a JEOL JCM-5000 Neoscope benchtop SEM (JEOL USA Inc., Peabody, MA) to observe any structural degradation of the materials.

Chemical changes in the material were observed with attenuated total reflectance Fourier transform infrared (ATR-FTIR) spectrometry. At day 0 and day 30 a thin slice $(2-3 \mathrm{~mm})$ was taken from a sacrificial sample and spectra were collected using Bruker ALPHA Infrared Spectrometer (Bruker, Billerica, MA). For each sample, 64 background scans were taken, followed by 32 scans of the sample. Spectra was collected in absorption mode with a resolution of $1 \mathrm{~cm}^{-1}$. OPUS software was used to perform baseline and atmospheric corrections. The intensities of sample peaks over time were compared by using the carbon skeletal peak at $1243 \mathrm{~cm}^{-1}$ as a baseline.

\subsection{Statistical Analysis}

All data are expressed as the mean \pm standard deviation of the mean. Statistical analysis was performed in JMP using unpaired Student's t-tests with $\mathrm{p}<0.05$ accepted as statistical significance. For gravimetric studies on the influence of aging on degradation, $N=8$ throughout all time points because sacrificial samples for SEM and FTIR were not included in weighing. For gravimetric studies on the influence of sterilization and composition on degradation, sacrificial samples were included in weighing until sacrifice at day 30 and day 60 . Thus, for these studies, $\mathrm{N}=8$ until day $30, \mathrm{~N}=7$ from days $36-60$, and $\mathrm{N}=6$ from day 66 until termination. 


\section{Results}

\subsection{Foam Characterization}

Prior to aging or sterilization treatment, pore size and density data were collected for all foams. For $\mathrm{H} 60$ foams, the average pore size was $217.7 \pm 99.8 \mu \mathrm{m}$ in the axial direction and $221.4 \pm 95.9 \mu \mathrm{m}$ in the transverse direction with an average density of $0.0378 \pm 0.0047 \mathrm{~g} \mathrm{~cm}^{-3}$. For $\mathrm{H} 70$ foams, the average pore size was $207.7 \pm 86.3 \mu \mathrm{m}$ in the axial direction and $187.5 \pm 73.7 \mu \mathrm{m}$ in the transverse direction with an average density of $0.0447 \pm 0.0067 \mathrm{~g} \mathrm{~cm}^{-3}$.

\subsection{Influence of Aging on Degradation}

We first investigated the influence of material ageing on the degradation profile of $\mathrm{H} 60$ foams sterilized at $25 \mathrm{kGy}$ when degraded in oxidative and hydrolytic conditions. Figure 2 shows the gravimetric degradation profile of aged and unaged foams in real-time and accelerated oxidative and hydrolytic media. These profiles align with previously seen results [11], showing significant degradation in an oxidative environment (Figure 2a) and negligible degradation in hydrolytic conditions (Figure 2b). Furthermore, a significant change in degradation profile is observed between aged and non-aged samples when degraded in a real-time $\left(3 \% \mathrm{H}_{2} \mathrm{O}_{2}\right)$ oxidative solution, but not when samples were degraded in an accelerated $\left(20 \% \mathrm{H}_{2} \mathrm{O}_{2}\right)$ solution.

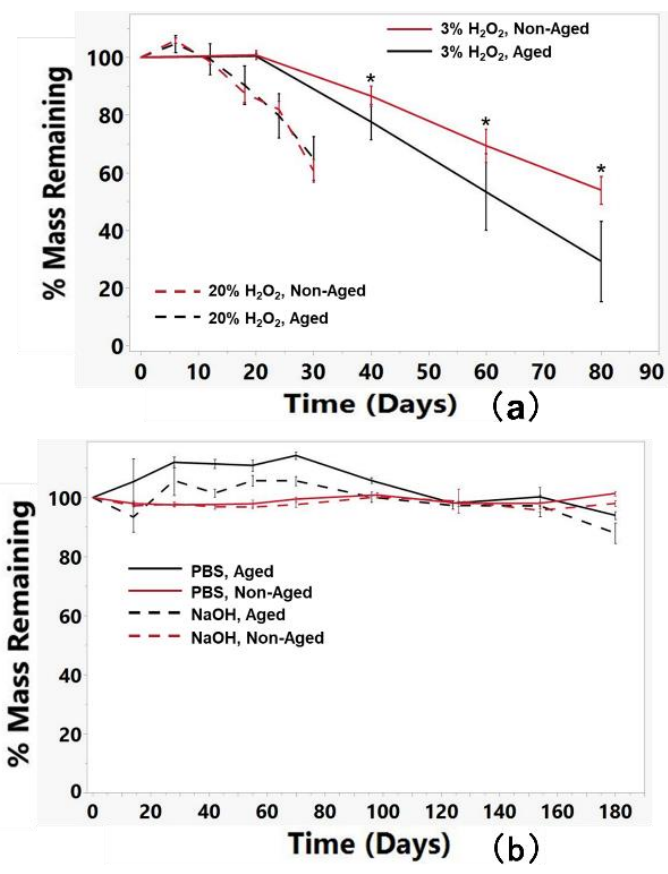

Figure 2 (a) Gravimetric degradation analysis for $\mathrm{H} 60$ foams in 3\% (real-time) and 20\% (accelerated) $\mathrm{H}_{2} \mathrm{O}_{2}$ solutions. Error bars show one standard deviation from the mean. * indicate statistical difference $(p<0.05)$ between aged and non-aged samples in $3 \% \mathrm{H}_{2} \mathrm{O}_{2}$ at the indicated time point. (b) Gravimetric degradation analysis for $\mathrm{H} 60$ foams in PBS (real-time) and $0.1 \mathrm{M} \mathrm{NaOH}$ (accelerated) hydrolytic solutions. Error bars show one standard deviation from the mean. 
FTIR spectra of the foams were collected throughout the degradation process to investigate chemical changes. Figure 3 shows the FTIR spectra of the aged and unaged foams when degraded oxidatively and hydrolytically. Figure 3a shows a shift in the urethane peak at $1692 \mathrm{~cm}^{-1}$ and a loss of the tertiary amine peak at $1052 \mathrm{~cm}^{-1}$ when samples are degraded in oxidative solutions. These changes in the FTIR spectra match previously observed phenomena [11] indicating a scission at the $\mathrm{C}-\mathrm{N}$ bond in the tertiary amines. Figure $3 \mathrm{~b}$ demonstrates a lack of chemical degradation in hydrolytic conditions with no observable changes in the FTIR spectra between days 0 and 30 . There were no notable differences in FTIR spectra between the aged and unaged samples for either oxidative or hydrolytic degradation.
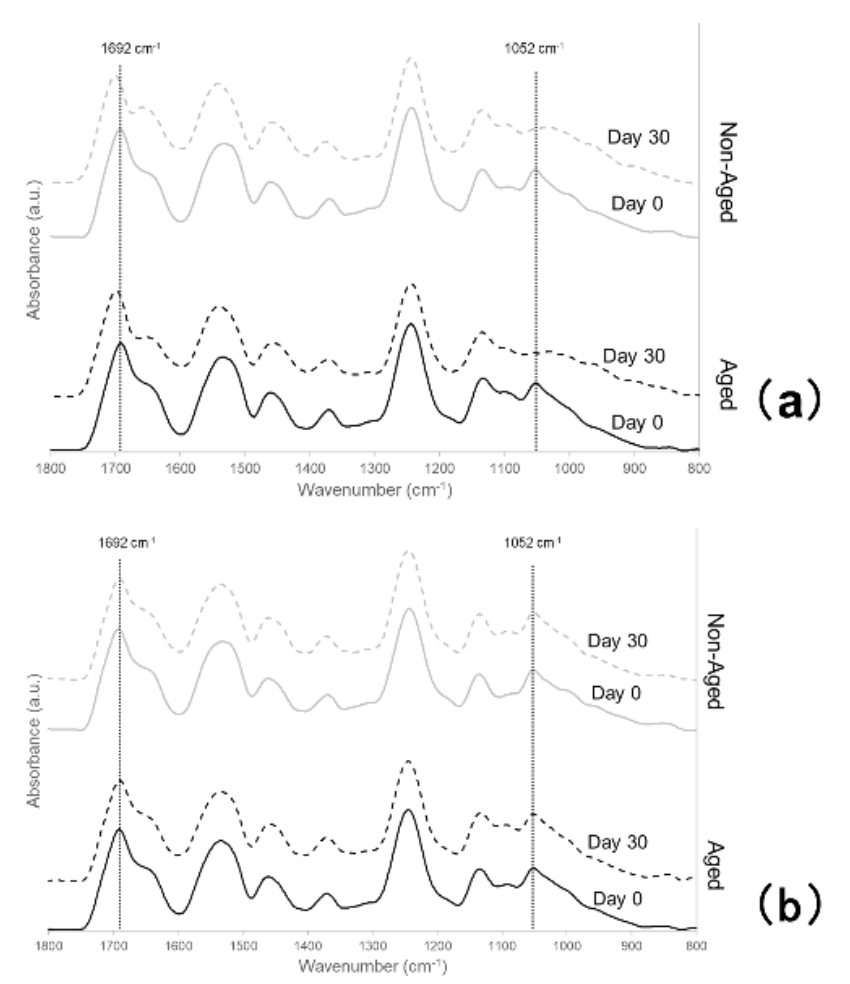

Figure 3 (a) FTIR analysis of oxidatively degraded $\mathrm{H} 60$ foams at day 0 and day 30 in $3 \%$ $\mathrm{H}_{2} \mathrm{O}_{2}$. The dotted line at $1692 \mathrm{~cm}^{-1}$ indicates the urethane peak of day 0 foams and shows the left shift of the urethane peak during degradation. The dotted line at $1052 \mathrm{~cm}^{-1}$ indicates the tertiary amine in day 0 foams which undergoes oxidative degradation. (b) FTIR analysis of hydrolytically degraded foams at day 0 and day 30 in $0.1 \mathrm{M} \mathrm{NaOH}$.

SEM images of the foams were also gathered during the degradation process to show morphological changes. Figure 4 provides SEM images of the foams at days 0 and 30 of the degradation process. In the oxidatively degraded samples, collapse of the porous structure can be observed at day 30 while no observable structure loss is seen in the hydrolytically degraded samples, further confirming the lack of degradation indicated by gravimetric and chemical studies. Similar morphological changes were observed in aged and non-aged samples. 


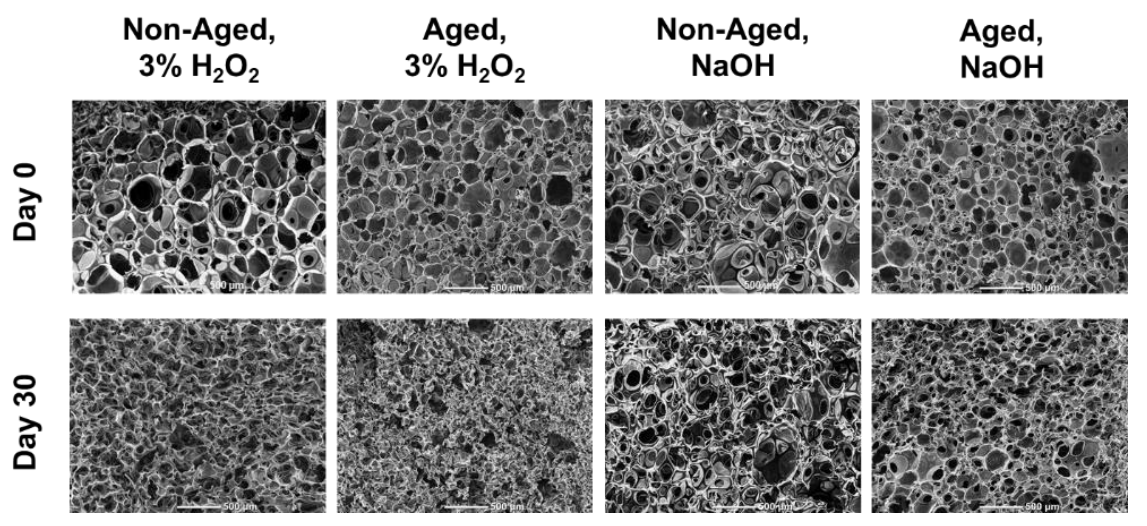

Figure 4 SEM images of oxidatively $\left(3 \% \mathrm{H}_{2} \mathrm{O}_{2}\right)$ and hydrolytically $(0.1 \mathrm{M} \mathrm{NaOH})$ degraded H60 foams at day 0 and day 30. Loss of strut integrity is observed for both aged and nonaged foams after oxidative degradation while strut integrity is preserved in hydrolytically degraded foams.

\subsection{Influence of Sterilization and Composition on Degradation}

The next study investigated the influence of high and low E-beam irradiation dosage levels of on the degradation profile of two foam compositions ( $\mathrm{H} 60$ and $\mathrm{H} 70$ ). All degradation was performed in an accelerated oxidative solution $\left(20 \% \mathrm{H}_{2} \mathrm{O}_{2}\right)$. Figure 5 plots the gravimetric degradation profile of the foams where no significant differences were observed between electron beam dosage or foam composition groups.

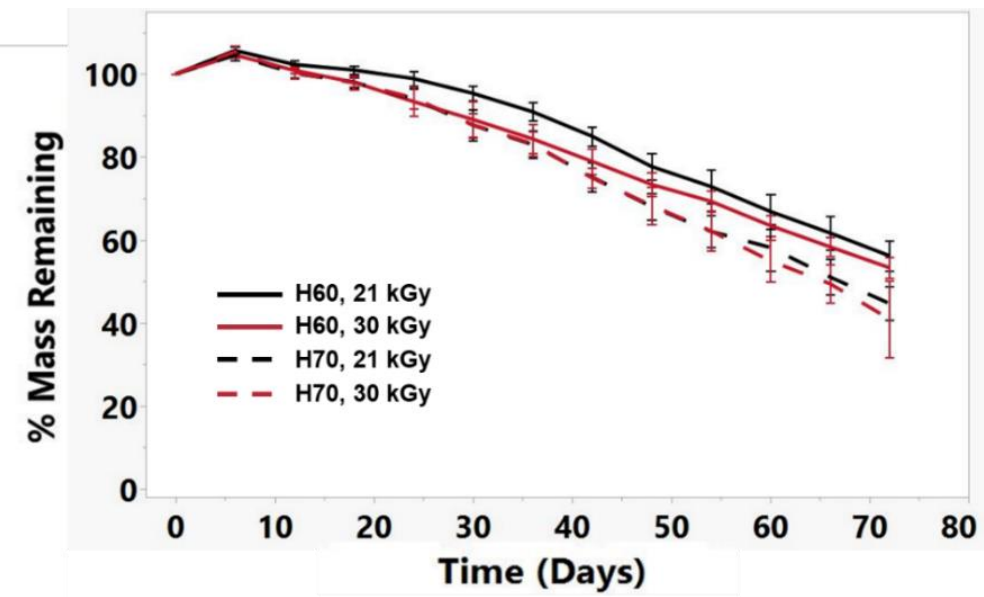

Figure 5 Gravimetric degradation analysis for $\mathrm{H} 60$ and $\mathrm{H} 70$ foams sterilized at 21 or 30 $\mathrm{kGy}$ in $20 \% \mathrm{H}_{2} \mathrm{O}_{2}$ solutions. Error bars show one standard deviation from the mean.

Figure 6 shows the FTIR spectra for these foams demonstrating the same left shift of the urethane peak and loss of the tertiary amine peak when degraded oxidatively. However, there are no observable differences between samples treated with different e-beam dosages, supporting the observations of the gravimetric degradation. 


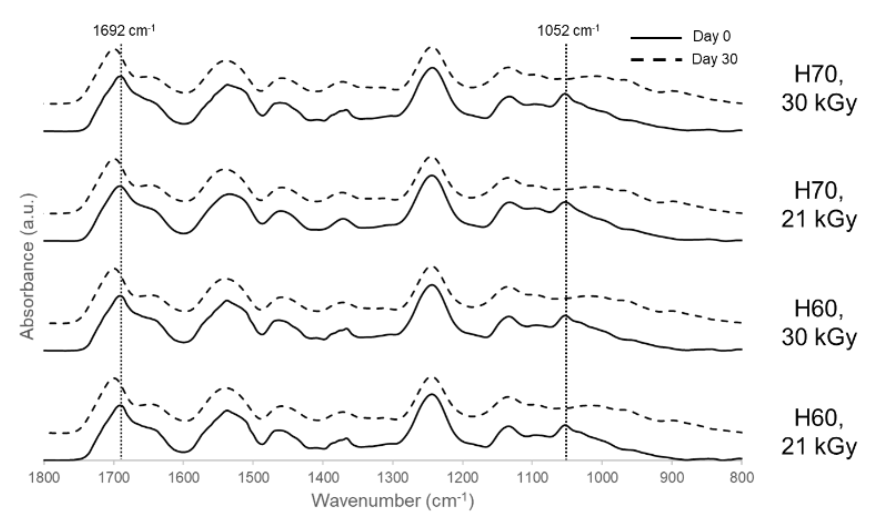

Figure 6 FTIR analysis of sterilized ( 21 or $30 \mathrm{kGy}$ ), oxidatively degraded foams at day 0 and day 30 in $3 \% \mathrm{H}_{2} \mathrm{O}_{2}$. A shift in the urethane peak at $1692 \mathrm{~cm}^{-1}$ and loss of the tertiary amine at $1052 \mathrm{~cm}^{-1}$ is observed for all compositions and sterilizations.

SEM images of these foams (Figure 7) were collected on day 50 of the degradation process. Collapse of the porous structures was observed for all samples indicating significant mass loss with no notable differences between sterilization dosage groups.

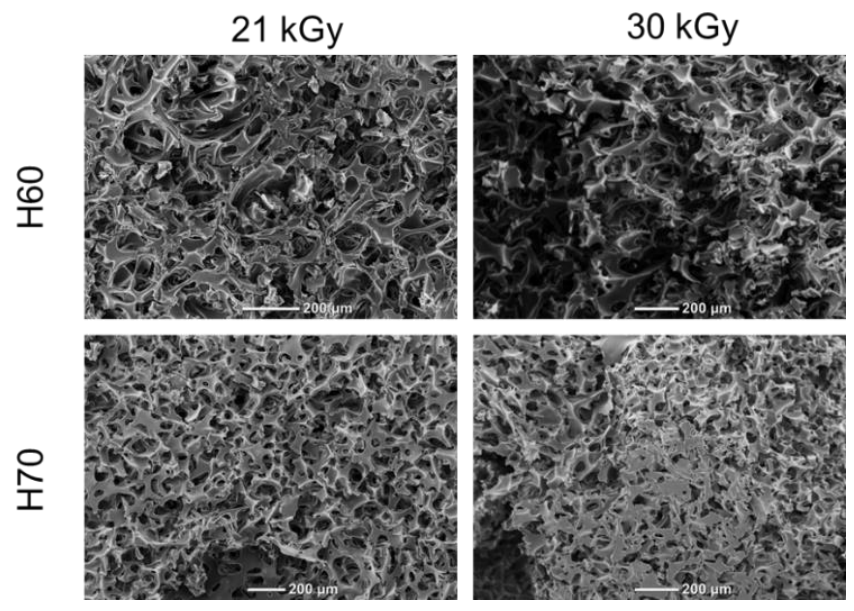

Figure 7 SEM images of oxidatively degraded foams at day 50. Similar strut loss is observed for foams with different compositions and treated with different sterilizations.

\section{Discussion}

The aim of this study was to investigate how aging, sterilization, and chemical composition may influence the degradation of SMP foams. Gravimetric studies showed the overall mass loss of materials in various solutions while ATR-FTIR readings showed chemical changes to the material backbone when degraded oxidatively. Finally, SEM images captured the morphological changes of the material during various stages of degradation. Together, these studies further inform appropriate biomedical applications of SMP foams while accounting for shelf life and sterilization parameters.

Aging studies showed that oxidative degradation does appear to occur more quickly in real time solutions when the materials are aged. Previous studies have demonstrated that thermal aging can decrease the amount of hydrogen bonding in polyurethane foams, leading to increased 
susceptibility to water penetration [37]. This data may explain why the aged samples degraded faster than the non-aged samples in the real time oxidative studies. Notably, this was not seen in the accelerated studies where the rate of degradation was likely less dependent on aqueous penetration into the polymer system, indicating the importance of real time degradation studies in biomaterial characterization. A more thorough understanding of the cause of this change in degradation rate will be important for future testing of devices, and it may inform which tests require aging to provide accurate and reliable results.

Sterilization studies investigated the high and low ranges of commonly used E-beam irradiation dosages and found no significant influence on accelerated oxidative degradation profile for either foam composition tested. This suggests that these dosages do not initiate chemical changes or alter the degradation rate of these materials. It is likely that if significantly higher dosages were used, such changes could occur [19], but such dosages would be outside those commonly used for sterilizing medical devices. This is an important finding for the commercial application of SMP foams because it provides flexibility in the sterilization process without concern for potential influences on the degradation profile. It also shows that sterilization does not influence various foam compositions differently, indicating they can safely be tuned to allow for appropriate working times or stiffness. It was observed that the $\mathrm{H} 70$ foams degraded slightly faster than $\mathrm{H} 60$ foams. Previous work with these foams found that the tertiary amines in TEA and HPED are susceptible to oxidative degradation and laid out a proposed degradation pathway for these compounds [11]. Because H70 foams have a higher molar concentration of HPDE, which has two susceptible tertiary amines, it is expected that they would undergo slightly faster oxidative degradation. Future studies will focus on real-time degradation analysis to expand upon this data. However, the degradation studies correlate with our previous work that shows that high dosages of E-beam irradiation (40 kGy) do not significantly impact SMP foam expansion, thermal, or mechanical properties [14].

Of note, in all gravimetric studies foams had recorded weights above $100 \%$ at the early time points. This is due to residual solvent (water) that is not completely removed during the drying process. It was observed that all solvent could be removed by drying the foams at $100{ }^{\circ} \mathrm{C}$, however, this is above the Tg of these materials and thus was not performed because it would have caused additional thermal degradation. Additionally, the degradation rate for $\mathrm{H} 60$ foams did differ significantly between the studies presented in Figures 2 and 5 . While the same protocols were followed for both, other work with these foams has shown that slight differences in handling (collection of particles during drying, removal of foams from the vial, foam compression), foam synthesis (reticulation of the foams, starting foam weights), oven temperatures, and $\mathrm{H}_{2} \mathrm{O}_{2}$ Concentration can cumulate is varying degradation rates. However, the goal of these studies was to determine the relative influence of various treatments (aging, sterilization, composition) on the degradation rate, not to determine the in vivo degradation rate of these material as that has been previously studied [11]. For this reason, conclusions for this paper were drawn only based on data within each study and we caution against drawing any conclusion between studies.

In addition to the understanding provided by these studies, further studies investigating the mechanical properties of foams after aging or sterilization and during degradation would be informative. Previous work investigating the mechanical properties of similar foams [38] showed that while the tensile strength decreases over time, samples were robust enough to test after 2.5 months of accelerated degradation. 


\section{Conclusions}

In summary, these studies demonstrated an influence, or lack thereof, of ageing, sterilization, and foam composition on the degradation profile of shape memory polymers intended for biomedical applications. Of note, we demonstrated that e-beam sterilization dosages between 21 and $30 \mathrm{kGy}$ do not influence degradation profile of these polyurethane foams. The influence of accelerated aging was not observed in accelerated oxidative degradation studies but could be seen in real-time oxidative studies. Slight differences in degradation rate for $\mathrm{H} 60$ and $\mathrm{H} 70$ foams were observed as expected, but neither were affected by e-beam sterilization. These studies will inform the processing and application of these materials in medical devices.

\section{Acknowledgments}

The authors would like to thank Dr. Landon Nash and Dr. Lindy Jang for their assistance in creating a representative schematic of the chemical structure for these materials.

\section{Author Contributions}

Sam Briggs: Gravimetric, FTIR, and SEM studies, data analysis, manuscript preparation. Mary Beth B Monroe: Gravimetric, FTIR, and SEM studies, manuscript edits. Mark Wierzbicki: Gravimetric, FTIR and SEM studies, manuscript edits. Sayyeda Marziya Hasan: Foam synthesis, sterilization, and aging, manuscript edits. Duncan J. Maitland: Experimental concept, supervision, funding acquisition, manuscript preparation.

\section{Funding}

This work was supported by the NIH National Institute of Neurological Disorder and Stroke grant U01-NS089692. This project was also supported in part by funding from Shape Memory Medical, Inc.

\section{Competing Interests}

Dr. Marziya Hasan, Dr. Mark Wierzbicki, and Dr. Duncan Maitland hold stock in the company, Shape Memory Medical, Inc. (SMM) (Santa Clara, CA, shapemem.com), that is commercializing the shape memory polymer foam technology featured in this manuscript. Further, Dr. Marziya Hasan is currently employed at SMM and Dr. Maitland is a co-founder, Chief Technology Officer and board member.

\section{References}

1. Chang JK, Emon MA, Li CS, Yang QS, Chang HP, Yang ZJ, et al. Cytotoxicity and in vitro degradation kinetics of foundry-compatible semiconductor nanomembranes and electronic microcomponents. ACS Nano. 2018; 12: 9721-9732.

2. Costa A, Naranjo JD, Turner NJ, Swinehart IT, Kolich BD, Shaffiey SA, et al. Mechanical strength vs. degradation of a biologically-derived surgical mesh over time in a rodent full thickness abdominal wall defect. Biomaterials. 2016; 108: 81-90. 
3. Santerre JP, Woodhouse K, Laroche G, Labow RS. Understanding the biodegradation of polyurethanes: From classical implants to tissue engineering materials. Biomaterials. 2005; 26: 7457-7470.

4. Coury AJ, Slaikeu PC, Cahalan PT, Stokes KB, Hobot CM. Factors and interactions affecting the performance of polyurethane elastomers in medical devices. J Biomater Appl. 1988; 3: 130-179.

5. Ahmed N, Kausar A, Muhammad B. Advances in shape memory polyurethanes and composites: A review. Polym-Plast Technol Eng. 2015; 54: 1410-1423.

6. Meng $\mathrm{Q}$, Hu J. A review of shape memory polymer composites and blends. Compos Part Appl Sci Manuf. 2009; 40: 1661-1672.

7. Peterson GI, Dobrynin AV, Becker ML. Biodegradable shape memory polymers in medicine. Adv Healthc Mater. 2017; 6: 1700694.

8. Xia YL, He Y, Zhang FH, Liu YJ, Leng JS. A review of shape memory polymers and composites: Mechanisms, materials, and applications. Adv Mater. 2021; 33: 2000713.

9. Kong DY, Li J, Guo A, Yu JX, Xiao XL. Smart polyimide with recovery stress at the level of high temperature shape memory alloys. Smart Mater Struct. 2021; 30: 035027.

10. Kong DY, Li J, Guo A, Xiao XL. High temperature electromagnetic shielding shape memory polymer composite. Chem Eng J. 2021; 408: 127365.

11. Weems AC, Wacker KT, Carrow JK, Boyle AJ, Maitland DJ. Shape memory polyurethanes with oxidation-induced degradation: In vivo and in vitro correlations for endovascular material applications. Acta Biomater. 2017; 59: 33-44.

12. Hasan SM, Fletcher GK, Monroe MB, Wierzbicki MA, Nash LD, Maitland DJ. Shape memory polymer foams synthesized using glycerol and hexanetriol for enhanced degradation resistance. Polymers. 2020; 12: 2290.

13. Jang LK, Fletcher GK, Monroe MB, Maitland DJ. Biodegradable shape memory polymer foams with appropriate thermal properties for hemostatic applications. J Biomed Mater Res A. 2020; 108: 1281-1294.

14. Muschalek R, Nash L, Jones R, Hasan SM, Keller BK, Monroe MB, et al. Effects of sterilization on shape memory polyurethane embolic foam devices. J Med Devices. 2017; 11: 031011-031019.

15. Premnath V, Harris WH, Jasty M, Merrill EW. Gamma sterilization of UHMWPE articular implants: An analysis of the oxidation problem. Biomaterials. 1996; 17: 1741-1753.

16. Benson RS. Use of radiation in biomaterials science. Nucl Instrum Methods Phys Res Sect B. 2002; 191: 752-757.

17. Adem E, Angulo-Cervera E, González-Jiménez A, Valentín JL, Marcos-Fernández A. Effect of dose and temperature on the physical properties of an aliphatic thermoplastic polyurethane irradiated with an electron beam. Radiat Phys Chem. 2015; 112: 61-70.

18. Bruyas A, Moeinzadeh S, Kim S, Lowenberg DW, Yang YP. Effect of electron beam sterilization on three-dimensional-printed polycaprolactone/beta-tricalcium phosphate scaffolds for bone tissue engineering. Tissue Eng Part A. 2018; 25: 248-256.

19. Murray KA, Kennedy JE, McEvoy B, Vrain O, Ryan D, Cowman R, et al. The influence of electron beam irradiation conducted in air on the thermal, chemical, structural and surface properties of medical grade polyurethane. Eur Polym J. 2013; 49: 1782-1795.

20. Kang JH, Kaneda J, Jang JG, Sakthiabirami K, Lui E, Kim C, et al. The influence of electron beam sterilization on in vivo degradation of $\beta-T C P / P C L$ of different composite ratios for bone tissue engineering. Micromachines. 2020; 11: 273. 
21. Kiliaris $P$, Papaspyrides CD, Pfaendner R. Influence of accelerated aging on clay-reinforced polyamide 6. Polym Degrad Stab. 2009; 94: 389-396.

22. Lesaffre $N$, Bellayer S, Fontaine $G$, Jimenez $M$, Bourbigot $S$. Revealing the impact of ageing on a flame retarded PLA. Polym Degrad Stab. 2016; 127: 88-97.

23. Liszkowska J, Moraczewski K, Borowicz M, Paciorek-Sadowska J, Czupryński B, Isbrandt M. The effect of accelerated aging conditions on the properties of rigid polyurethane-polyisocyanurate foams modified by cinnamon extract. Appl Sci. 2019; 9: 2663.

24. Oliani WL, Komatsu LG, Lugao AB, Parra DF. Thermal ageing and accelerated weathering of HMSPP: Structural and morphological studies. Macromol Symp. 2016; 367: 18-23.

25. Panaitescu I, Koch T, Archodoulaki VM. Accelerated aging of a glass fiber/polyurethane composite for automotive applications. Polym Test. 2019; 74: 245-256.

26. Madej-Kiełbik L, Kośla K, Zielińska D, Chmal-Fudali E, Maciejewska M. Effect of accelerated ageing on the mechanical and structural properties of the material system used in protectors. Polymers. 2019; 11: 1263.

27. Mahomed A, Hukins DW, Kukureka SN. Effect of accelerated aging on the viscoelastic properties of a medical grade silicone. Biomed Mater Eng. 2015; 25: 415-423.

28. de Souza Rios A, de Amorim WF, de Moura EP, de Deus EP, de Andrade Feitosa JP. Effects of accelerated aging on mechanical, thermal and morphological behavior of polyurethane/epoxy/fiberglass composites. Polym Test. 2016; 50: 152-163.

29. Boubakri A, Haddar N, Elleuch K, Bienvenu Y. Impact of aging conditions on mechanical properties of thermoplastic polyurethane. Mater Des. 2010; 31: 4194-4201.

30. Boubakri A, Haddar N, Elleuch K, Bienvenu Y. Influence of thermal aging on tensile and creep behavior of thermoplastic polyurethane. Comptes Rendus Mécanique. 2011; 339: 666-673.

31. Khan AA, Al-Kheraif AA, Al-Shehri AM, Säilynoja E, Vallittu PK. Polymer matrix of fiber-reinforced composites: Changes in the semi-interpenetrating polymer network during the shelf life. J Mech Behav Biomed Mater. 2018; 78: 414-419.

32. Xin H, Shepherd DE, Dearn KD. Strength of poly-ether-ether-ketone: Effects of sterilisation and thermal ageing. Polym Test. 2013; 32: 1001-1005.

33. Yarahmadi N, Vega A, Jakubowicz I. Accelerated ageing and degradation characteristics of rigid polyurethane foam. Polym Degrad Stab. 2017; 138: 192-200.

34. Lambert BJ, Tang FW. Rationale for practical medical device accelerated aging programs in AAMI TIR 17. Radiat Phys Chem. 2000; 57: 349-353.

35. Weems AC, Boyle AJ, Maitland DJ. Two-year performance study of porous, thermoset, shape memory polyurethanes intended for vascular medical devices. Smart Mater Struct. 2017; 26: 035054.

36. Hasan SM, Raymond JE, Wilson TS, Keller BK, Maitland DJ. Effects of isophorone diisocyanate on the thermal and mechanical properties of shape-memory polyurethane foams. Macromol Chem Phys. 2014; 215: 2420-2429.

37. Zieleniewska M, Leszczyński MK, Szczepkowski L, Bryśkiewicz A, Krzyżowska M, Bień K, et al. Development and applicational evaluation of the rigid polyurethane foam composites with egg shell waste. Polym Degrad Stab. 2016; 132: 78-86.

38. Weems AC, Wacker KT, Maitland DJ. Improved oxidative biostability of porous shape memory polymers by substituting triethanolamine for glycerol. J Appl Polym Sci. 2019; 136: 47857. 


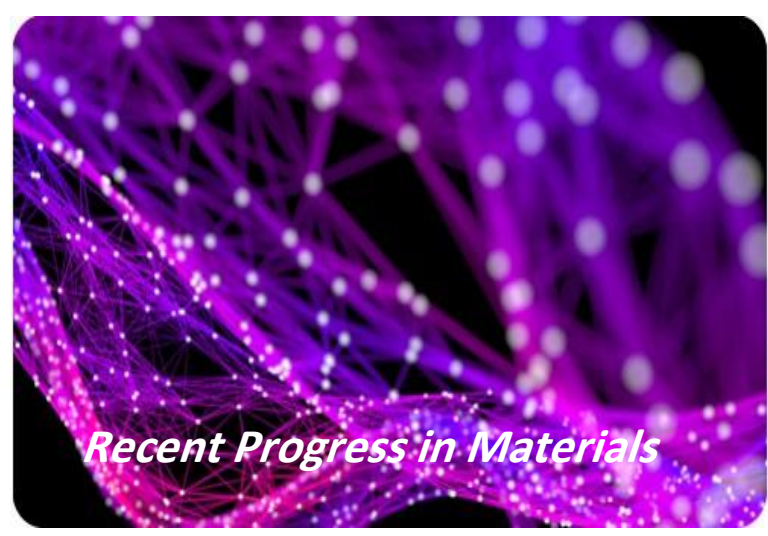

Enjoy Recent Progress in Materials by:

1. Submitting a manuscript

2. Joining in volunteer reviewer bank

3. Joining Editorial Board

4. Guest editing a special issue

For more details, please visit:

http://www.lidsen.com/journals/rpm 